Copyright (c) 2005 IEEE. Reprinted from

Guangran Zhu, Brent R. Petersen and Bruce G. Colpitts, "Signalling wavelength in an antenna array for space-time wireless over LOS channels," IEEE Proceedings of the 3rd Annual Communications Networks Services and Research Conference (CNSR 2005), vol. 1, (Halifax, NS, Canada), pp. 69-73, May 16-18. 2005.

This material is posted here with permission of the IEEE. Internal or personal use of this material is permitted. However, permission to reprint/republish this material for advertising or promotional purposes or for creating new collective works for resale or redistribution must be obtained from the IEEE by sending a blank email message to pubs-permissions@ieee.org.

By choosing to view this document, you agree to all provisions of the copyright laws protecting it. 
(CNSR 2005) vol. 1, (Halifax, NS, Canada), pp. 69-73, May 16-18, 2005.

\title{
Signalling Wavelength in an Antenna Array for Space-Time Wireless over LOS Channels
}

\author{
Guangran Zhu, Brent R. Petersen, and Bruce G. Colpitts \\ University of New Brunswick \\ Dept. of Electrical and Computer Engineering \\ Fredericton, NB, Canada, E3B 5A3 \\ E-mail: \{kevin.zhu,colpitts\}@unb.ca,b.petersen@ieee.org
}

\begin{abstract}
We consider the inter-antenna separation in a distributed antenna array. We introduce a new separation constraint with respect to the signalling wavelength, which equals the chip wavelength in DS-CDMA systems and the symbol wavelength in non-spread systems. This constraint ensures the phases of the frequency responses are exercised over $2 \pi$ in the baseband channel matrix. The diverse phases provide a better channel matrix for signal detection. The multiuser performance improvement due to this constraint has been named as the Signalling Wavelength Antenna Placement (SWAP) gain.
\end{abstract}

\section{Introduction}

Space-Time (ST) technology provides a powerful method for improving the performance of wireless systems. A ST receiver uses multiple antennas for spatial diversity and uses temporal filters to process the signal at each antenna branch. When all users occupy the same bandwidth at the same time, the ST receiver can be regarded as an equalizer which effectively inverts the channel matrix at baseband [1] to recover the transmitted signals of individual users. The invertibility of the baseband channel matrix affects the performance of the ST receiver.

Lee [2] shows low signal envelope correlation when two receiving antennas are separated by half a carrier wavelength in a rich scattering environment. This assumption is widely adopted, and the study of correlated fading has been focused on antenna separations on the scale of the carrier wavelength. In the meanwhile, the studies of ST receivers, e.g. [3] usually assume independent fading at each receiving antenna. This implies that the magnitude of the frequency response of each path is different and the complex baseband channel matrix is easy to invert. However, it is possible to observe very high envelope correlation when antennas are separated as far as hundreds of carrier wavelengths, e.g. a
Local Multipoint Communication System (LMCS) [4]. In such a case, the wireless environment usually has a LOS component with few scatters. The magnitude of the frequency response of each path is almost identical.

This paper studies the invertibility of the channel matrix in a LOS environment in which all paths have similar frequency response but are differed by a phase offset due to the antenna separation. Analytical and computer simulation results show that an inter-antenna separation comparable to the signalling wavelength improves the invertibility of the baseband channel matrix more than a separation comparable to the carrier wavelength. A similar concept of the signaling wavelength has been reported in [5].

Section II presents the system and channel model. Section III presents the analysis for a two by two channel matrix. Section IV presents the simulation results, and Section V the conclusions.

\section{System and Channel model}

Fig. 1 depicts the system model. We consider the uplink of a wireless communication system in a multiuser environment. M portables, each with one antenna, are transmitting to $\mathrm{N}$ receiving antenna array connected to a ST receiver. The paths between the portables and the receiving antennas are LOS and without mulitpath effect. The distance from portable $m$ to receiving antenna $n$ is $l_{m n}$, and the delay is $l_{\mathrm{mn}} / \mathrm{c}=\tau_{\mathrm{mn}}$, where $\mathrm{c}$ is the propagation velocity. All paths have a total bandwidth $f_{\mathrm{g}}$, which is also denoted as the signalling rate in this paper.

\section{Analytical Results}

Since we are only concerned with the inter-antenna separation, our discussion is around the simplest nontrivial system including two portables and an array with two receiving antennas, i.e. $\mathrm{M}=\mathrm{N}=2$. When two receiving antennas are located very close, the frequency response of the paths between one portable and the two receiving antennas are almost identical. However, there exists a 
phase offset due to the difference in the time delay between the two paths. This is shown in Fig. 2 (a), and the two by two complex baseband channel matrix is

$$
\Phi=\left[\begin{array}{ll}
A(f) e^{j \theta_{A}(f)} & A(f) e^{j \theta_{A}(f)-2 \pi f\left(\tau_{11}-\tau_{12}\right)} \\
B(f) e^{j \theta_{B}(f)} & B(f) e^{j \theta_{B}(f)-2 \pi f\left(\tau_{21}-\tau_{22}\right)}
\end{array}\right],
$$

where $A(f)$ and $B(f)$ denote the different magnitude responses from two portables, $\theta_{A}(f)$ and $\theta_{B}(f)$ denote the phase responses, and the baseband frequency is

$$
f \in\left[-\frac{1}{2} f_{g},+\frac{1}{2} f_{g}\right]
$$

The phase offsets of the first and the second portable are

$$
\beta_{1}(f)=2 \pi\left(\tau_{11}-\tau_{12}\right) f
$$

and

$$
\beta_{2}(f)=2 \pi\left(\tau_{21}-\tau_{22}\right) f
$$

The ranges of the phase offsets, as determined by the frequency (2), are

$$
\left|2 \pi\left(\tau_{11}-\tau_{12}\right) \frac{1}{2} f_{g}\right|>\beta_{1}(f)
$$

and

$$
\left|2 \pi\left(\tau_{21}-\tau_{22}\right) \frac{1}{2} f_{g}\right|>\beta_{2}(f) .
$$

If the phase offsets are limited to a small range, we get values close to zero over the frequency and the channel matrix is hard to invert. The maximum meaningful range for the phase offsets is $[-\pi,+\pi]$. In order to fully exercise over this range, we have

$$
\left|2 \pi\left(\tau_{11}-\tau_{12}\right) \frac{1}{2} f_{g}\right|>\pi
$$

and

$$
\left|2 \pi\left(\tau_{21}-\tau_{22}\right) \frac{1}{2} f_{g}\right|>\pi
$$

Applying the relations $\tau_{\mathrm{mn}}=l_{\mathrm{mn}} / \mathrm{c}$ and $f_{\mathrm{g}}=\mathrm{c} / \lambda_{\mathrm{g}}$ in (7) and (8), we get

$$
\left|l_{11}-l_{12}\right|>\lambda_{g}
$$

and

$$
\left|l_{21}-l_{22}\right|>\lambda_{g}
$$

The exact value of the path length differences $\left(l_{11}-l_{12}\right)$ and $\left(l_{21}-l_{22}\right)$ are dependent on specific portable locations. Only when the portables are close to the end-fire region of the array do the path length differences approach the antenna separation, $\Delta$. In general, they are limited within

$$
\left|l_{11}-l_{12}\right|<\Delta
$$

and

$$
\left|l_{21}-l_{22}\right|<\Delta \text {. }
$$

It is impractical to constrain all portables to the end-fire region in order to satisfy (9) and (10). However, the antenna separation greater than a signalling wavelength, at least gives the freedom to some locations, at which the phase offsets can fully exercise over $[-\pi,+\pi]$. This constraint is expressed as

$$
\Delta>\lambda_{g}
$$

This is our basic result.

In addition to having large phase offsets, we also need differing phase offset slopes, $2 \pi\left(\tau_{11^{-}} \tau_{12}\right)$ and $2 \pi\left(\tau_{21^{-}}\right.$ $\left.\tau_{22}\right)$ in (3) and (4) in order to ensure the invertibility of the channel matrix. If these offset slopes are identical, regardless of the range of the phase offsets for the two portables, their values are identical over the frequency range. The channel matrix remains singular. The offset slopes depend on the actual portable locations. We get identical offset slopes when the portables are very close to each other, or when the portables are in even symmetric locations with respect to the receiving antennas as shown in Fig. 2 (b), or when the receiving antennas are in even symmetric locations with respect to the portables as shown in Fig. 2 (c). Fig. 3 shows the phase offsets with similar and different offset slopes.

In this paper, we assume the portables are randomly distributed over a circular region. It is unlikely to get into these pathological cases. Given the condition that the phase offset can be fully exercised over $[-\pi,+\pi]$ and the assumption that the offset slopes are generally different, the channel matrix is easy to invert for most portable locations. 
Two things need to be pointed out. Since the phase offset is a linear function of the baseband frequency. When the baseband frequency becomes zero, so do the phase offsets and the channel matrix becomes singular. Also, there will be no advantage to have antenna separation greater than the signalling wavelength, because of the cyclic property of phase over $2 \pi$. It is also difficult to connect the antennas separated by a large distance. The signalling rate corresponds to the chip rate in DS-CDMA systems or symbol rate in non-spread systems. Antenna separations comparable to the signalling wavelength appear impractical to non-spread systems, however, as technology is pushing up the chip rate in DS-CDMA systems, our constraint (13) becomes realistic.

\section{Simulation Results}

In our simulation, the channel is assumed pure-delay so we can observe how the antenna separation affects the invertibility of the channel matrix without being concerned with the magnitude of the frequency response. The invertibilty is evaluated by the 2-norm condition number. In the case of two portables and two receiving antennas, the channel matrix becomes

$$
\Phi=\left[\begin{array}{ll}
e^{-j 2 \pi f \tau_{11}} & e^{-j 2 \pi f \tau_{12}} \\
e^{-j 2 \pi f \tau_{21}} & e^{-j 2 \pi f \tau_{22}}
\end{array}\right]
$$

Fig. 4 shows the effect of the antenna separation on the $\log$ of the condition numbers at two random portable locations. The frequency, normalized with respect to the signalling frequency, is evaluated in the interval $[-1 / 2$, $+1 / 2]$. We can see that an increase in the antenna separation from $0.005 \lambda_{\mathrm{g}}$ to $\lambda_{\mathrm{g}}$ drastically improves the condition number, while there is marginal improvement as the separation increases to $5 \lambda_{\mathrm{g}}$. The peak at the center corresponds to the case when the baseband frequency is zero.

In the next simulation, 1000 sets of two portable locations are randomly distributed over a circular region with radius $200 \lambda_{\mathrm{g}}$. The average of the $\log$ of the condition numbers is calculated over the normalized frequency interval for each set of portable locations. The mean of the 1000 sets is then calculated and plotted for a particular antenna separation. Fig. 5 confirms that the mean of the condition number decreases as the antenna separation increases up to $\lambda_{\mathrm{g}}$ and there is a diminishing return as the antenna separation increases further.

We also make a comparison with the IEEE $802.11 \mathrm{~b}$ standard, which specifies a Direct Sequence Spread Spectrum (DSSS) system operating at $2.4 \mathrm{GHz}$ with a maximum chip rate at $11 \mathrm{MHz}$. The carrier wavelength is about $0.125 \mathrm{~m}$ and the chip wavelength is about $27.273 \mathrm{~m}$. The typical operating range outdoor is about $400 \mathrm{~m}$, corresponding to $14.667 \lambda_{\mathrm{g}}$. If two receiving antennas are separated at half carrier wavelength which is about $2.292 \times 10^{-3} \lambda_{\mathrm{g}}$, and two portables are randomly distributed within the operating range, the mean of the log of the condition numbers over 1000 trials is 3.437 . We obtain 0.8242 and 0.5787 when the receiving antennas are separated at $\lambda_{\mathrm{g}}$ and $5 \lambda_{\mathrm{g}}$.

\section{Conclusions and Future Work}

A new constraint on the inter-antenna separation of a distriubted antenna array in a highly correlated environment has been introduced. The antenna separation should be comparable with respect to the signalling wavelength and this gives varying phases in the baseband channel matrix. It is shown that this constraint significantly improves the invertibility of the channel matrix compared to the arrays whose antenna separation is on the scale of the carrier wavelength. It is expected the performance of ST receivers should be improved under this new constraint. This improvement has been named as the Signalling Wavelength Antenna Placement (SWAP) gain. This work can be useful to the forthcoming $802.11 \mathrm{n}$ MIMO standard.

\section{Acknowledgements}

This work was funded by the Atlantic Canada Opportunities Agency (ACOA) through the Atlantic Innovation Fund (AIF).
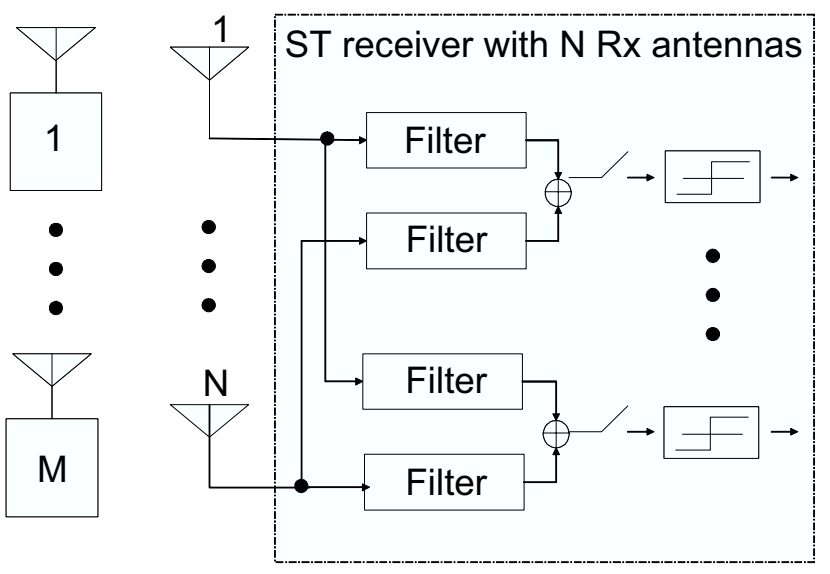

Figure 1. System model. 


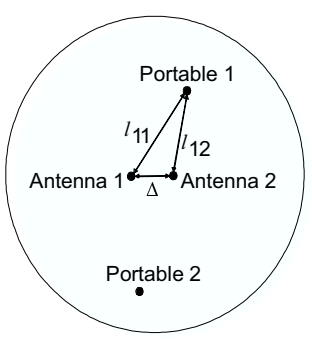

(a)

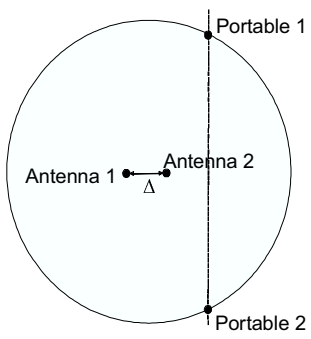

(b)

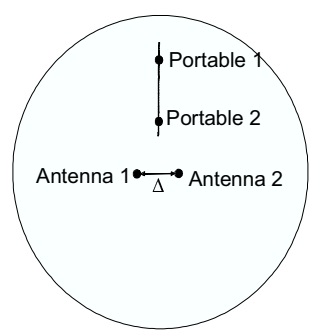

(c)

Figure 2. Two portable two receiving antenna scenarios (a) General case, (b) Symmetry w. r. t. Rx antennas, (c) Symmetry w. r. t. portables.

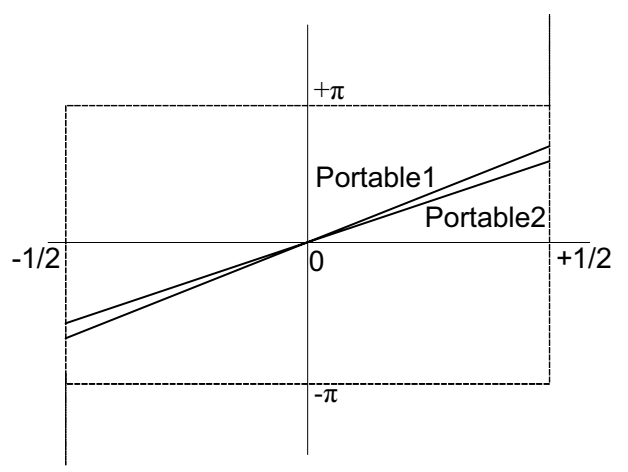

(a)

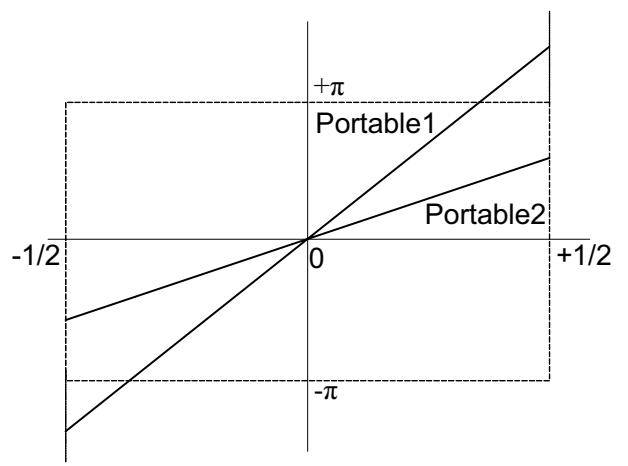

(b)

Figure 3. Phase offset versus normalized frequency (a) Phase offset slopes of the two portables are similar, (b) Phase offset slopes of the two portables are different, and Portable 1 may be at a location that has a phase range larger than $[-\pi,+\pi]$ if $\Delta$ is much greater than $\lambda_{\mathrm{g}}$.

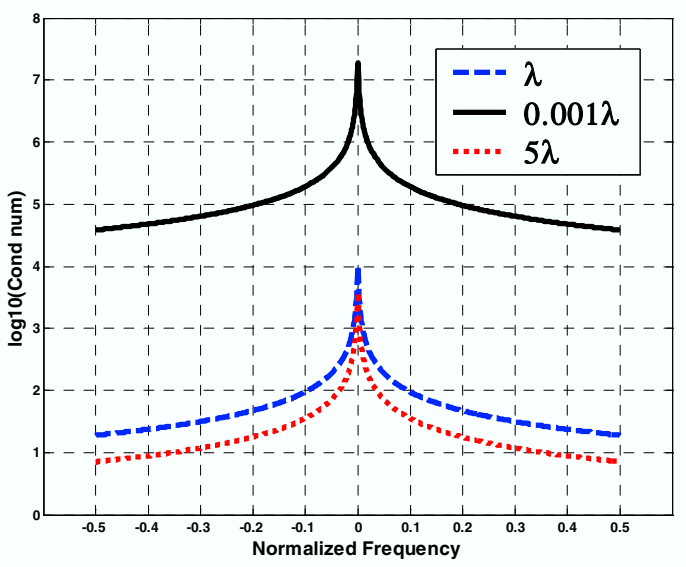

Figure 4. Condition numbers versus normalized frequency. Two portables are randomly located within a radius of $20 \lambda_{a}$.

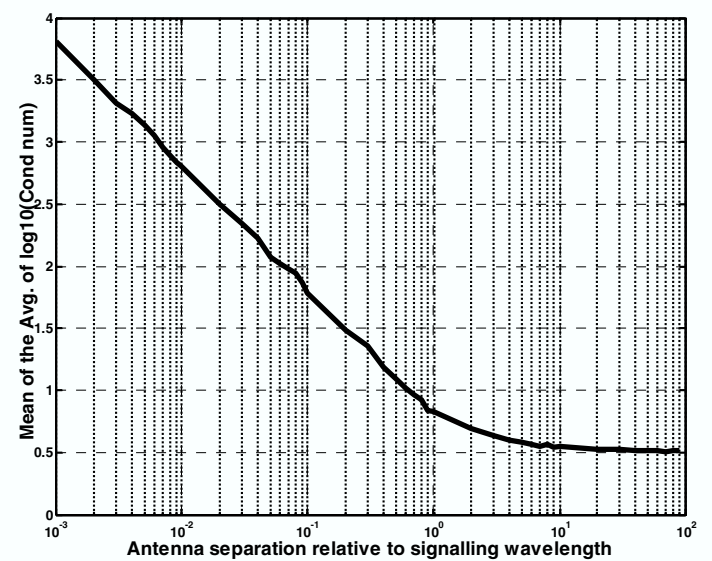

Figure 5. Mean of the average of the condition numbers versus antenna separation. Portables are randomly located within a radius of $200 \lambda_{\mathrm{g}}$.

\section{References}

[1] Brent R. Petersen, "Equalization in Cyclostationary Interference," Ph.D. thesis, Dept. of Systems and Computer Engineering, Carleton University, Ottawa, ON, Canada, 1992, ch. 3 .

[2] W. C. Y. Lee, "A study of the antenna array configuration of an M-branch diversity combining mobile radio receiver," IEEE Trans. Veh. Tech., vol. 20, pp. 93-104, Nov. 1971. 
[3] Ramon Schlagenhaufer, "Equalizer Structures for Spread Spectrum Multiuser Systems," Ph.D. thesis, Dept. of Electrical and Computer Engineering, University of Calgary, Calgary, AB, Canada, 2001, ch. 2.

[4] S. Roy and D. D. Falconer, "A three-dimensional wideband propagation model for the study of base station antenna arrays with application to LMCS," in pro. VTC'98, Ottawa, Canada, May 1998.

[5] H. Yanicomeroglu and E. S. Sousa, "Antenna gain against interference in CDMA macrodiversity systems," IEEE Trans. Commun., vol. 50, no. 8, pp. 1356-1371, Aug. 2002. 